\title{
Photoproduction of pions and etas in nuclei*
}

\author{
M. Effenberger, A. Hombach, S. Teis and U. Mosel \\ Institut für Theoretische Physik, Universität Giessen \\ Heinrich-Buff-Ring 16, D-35392 Giessen \\ UGI-96-22
}

\begin{abstract}
We calculate the cross sections for inclusive one-pion, two-pion and eta photoproduction in nuclei in the photon energy range from $300 \mathrm{MeV}$ to $900 \mathrm{MeV}$ within the framework of a semi-classical BUU transport model. Our results are compared with existing experimental data and discussed with respect to a calculation of the total photoabsorption cross section.
\end{abstract}

PACS numbers: 25.20.Lj, 25.20.x

Keywords: pion photoproduction, eta photoproduction, total photonuclear cross section, BUU transport model

\section{Introduction}

In a recent work [1] we have performed a calculation of the total photoabsorption cross section on nuclei within a semi-classical phase space model in order to understand the experimentally observed medium modifications compared to the elementary cross section on the proton and deuteron: the broadening of the $\Delta$-peak and the disappearance of the higher resonances $D_{13}$ and $F_{15}$ [2, 年, 专, 5, 6. We calculated the in-medium widths of the involved

*Work supported by BMBF and GSI Darmstadt 
nucleon resonances by using estimates for the cross sections for resonancenucleon scattering. It turned out that the collision widths, especially for the $D_{13}$, were much too small to explain the mentioned medium modifications; a collision width of $300 \mathrm{MeV}$ that has been ascribed to the $D_{13}$-resonance by phenomenological fits to the absorption cross section [7, 8, 9] seems far too large. In the $\Delta$-region the missing strength in the excitation function indicates that many-body absorption mechanisms should be included.

We now want to look at more exclusive reaction channels in order to gain further insight into the photon-nucleus reaction and have therefore calculated cross sections for one-pion, two-pion and eta production in nuclei. In these studies we have used a BUU transport model that employs the very same microscopic ingredients as the calculations in [1] and has been very successfully applied to the description of heavy-ion collisions up to bombarding energies of $2 \mathrm{GeV} / \mathrm{A}$ [10, 11, 12, 13] and pion-nucleus reactions [14]. We thus expect that the incoherent interaction of the primary produced mesons and nucleon resonances with the surrounding nuclear medium is quite well simulated. The non-perturbative treatment of all processes following the photon absorption makes the BUU model an excellent tool for these calculations. Therefore it should be possible to trace back medium modifications of the elementary photon-nucleon interaction.

Presently the total cross section for photoproduction of pions in nuclei has only been measured up to photon energies of $500 \mathrm{MeV}$ [15]. Theoretical calculations within the framework of the $\Delta$-hole model [16] and the coupled channel BUU model [17] were able to explain the size of the observed cross sections but failed in the description of the broad structure in the $\Delta$-region.

Pion photoproduction on nuclei is determined both by the elementary $(\gamma, \pi)$ process on the nucleon as well as by final state $\pi$ - $\mathrm{N}$ interactions whereas photoabsorption is dominated by the former reaction. A detailed investigation of $(\gamma, \pi)$ on nuclei could thus help to separate these two effects and to identify true in-medium effects on the primary production process. A possible medium modification might, for example, be a strong modification of the elementary two-pion production cross section or a lowering of the $\rho$-meson mass in nuclei 18 that would affect the width of the $N(1520)$-resonance.

In section 2 we start with a brief presentation of the used BUU model. The parameterizations of the elementary cross sections follow in section 3 which are used to calculate the photoproduction of pions (section 4.1) and etas (section 4.2). 


\section{The BUU model}

Since the BUU model has been described in full detail in the literature (e. g. [10, 11]) we restrict ourselves here to the basic ideas and focus on the points that are of particular interest for the calculations presented here. The BUU equation describes the classical time evolution of a many-particle system under the influence of a self-consistent mean field potential and a collision term. For the case of identical particles it is given by:

$$
\frac{\partial f}{\partial t}+\frac{\vec{p}}{m} \frac{\partial f}{\partial \vec{r}}-\vec{\nabla} U \frac{\partial f}{\partial \vec{p}}=I_{\text {coll }}[f]
$$

where $f(\vec{r}, \vec{p}, t)$ stands for the one-particle phasespace density, $U[f]$ denotes the self-consistent mean field potential and $I_{\text {coll }}[f]$ is the collision term which - for a fermionic system - respects the Pauli principle. For the description of a system of non-identical particles one gets an equation for each particle species that is coupled to all others by the collision integral or the mean field potential. Besides the nucleon we take all baryonic resonances up to a mass of $2 \mathrm{GeV}$ as well as the pion, the eta- and the rho-meson into ac-

count. Schematically one can write down the set of coupled equations in the following way:

$$
\begin{aligned}
D f_{N} & =F_{\text {coll }}^{N}\left(f_{N}, f_{\Delta(1232)}, f_{N(1440)}, \ldots, f_{\pi}, f_{\eta}, \ldots\right) \\
& =I_{N N}^{N}+I_{N \Delta}^{N}+I_{N N(1440)}^{N}+\ldots+I_{\Delta N \pi}^{N}+I_{N(1440) N \pi}^{N}+\ldots \\
D f_{\Delta(1232)} & =F_{\text {coll }}^{\Delta(1232)}\left(f_{N}, f_{\Delta(1232)}, f_{N(1440)}, \ldots, f_{\pi}\right) \\
D f_{N(1440)} & =F_{\text {coll }}^{N(1440)}\left(f_{N}, f_{\Delta(1232)}, f_{N(1440)}, \ldots, f_{\pi}, \ldots\right) \\
\ldots & \\
D f_{\pi} & =F_{\text {coll }}^{\pi}\left(f_{N}, f_{\Delta(1232)}, f_{N(1440)}, \ldots, f_{\pi}, \ldots\right) \\
D f_{\eta} & =F_{\text {coll }}^{\eta}\left(f_{N}, f_{N(1535)}, f_{\eta}\right) \\
\ldots &
\end{aligned}
$$

where $D$ abbreviates the operator of the Vlasov-equation given by the lhs of equation (11).

The initialization of $f_{N}$ for a nucleus at rest is done by using a WoodsSaxon type density distribution:

$$
\rho(r)=\rho_{0}\left[1+\mathrm{e}^{\frac{r-r_{0}}{\alpha}}\right]^{-1}
$$


with the following parameters:

$$
r_{0}=1.124 \mathrm{fm} A^{1 / 3} \quad \alpha=\left(0.024 A^{1 / 3}+0.29\right) \mathrm{fm} \quad .
$$

In momentum space we make use of a local Thomas-Fermi approximation. The local Fermi momentum $p_{F}(r)$ is thus given by:

$$
p_{F}(r)=\sqrt[3]{\frac{3}{2} \pi^{2} \rho(r)} .
$$

The mean field $U_{N}$ for the nucleons consists of a Skyrme and a Yukawa part and is described in [11]. This potential is also used for all isospin- $\frac{1}{2}$ resonances. For the $\Delta$-resonance we use a simple ansatz:

$$
U_{\Delta}(\rho)=U_{\Delta, 0} \frac{\rho}{\rho_{0}},
$$

with $U_{\Delta, 0}=-30 \mathrm{MeV}$ [19].

The collision term allows for the following reactions:

$$
\begin{aligned}
N N & \rightarrow N N \\
N N & \leftrightarrow N R \\
N N & \leftrightarrow N N \pi \quad(\mathrm{S} \text { - wave }) \\
N R & \rightarrow N R^{\prime} \\
R & \leftrightarrow N m \\
R & \leftrightarrow N \pi \pi \\
& \leftrightarrow \Delta(1232) \pi, N(1440) \pi, N \rho, N \sigma \\
\pi \pi & \leftrightarrow \rho, \sigma,
\end{aligned}
$$

where $\mathrm{R}$ stands for a baryonic resonance and $\mathrm{m}$ for a meson. The used decay widths and cross sections are described in detail in [1, 12].

\section{Parameterization of the elementary $\gamma N$ cross sections}




\subsection{One-pion production}

A coherent decomposition of the one-pion production cross sections into resonance and background contributions is necessary since - especially in the region of the $\Delta$-resonance - interference terms are quite important. Therefore the resonance contributions are not fitted to total cross sections but to partial-wave amplitudes $A_{l \pm}$ and $B_{l \pm}\left(j=l \pm \frac{1}{2}, l=\right.$ angular momentum of the $\pi N$-system) [20]. The amplitudes are taken from [21].

For the resonance amplitudes we make - following [20] - a Breit-Wigner ansatz under the assumption that the complex phase of the amplitude is equal to the phase of the resonance propagator for a spinless particle:

$$
\left\{\begin{array}{l}
A_{R}(\sqrt{s}) \\
B_{R}(\sqrt{s})
\end{array}\right\}=\left(\frac{k_{0} q_{0}}{k q}\right)^{1 / 2}\left(\frac{\Gamma_{0}}{\Gamma_{\pi}\left(M_{R}\right)}\right)^{1 / 2} \frac{\sqrt{s} \Gamma_{\pi}^{1 / 2} \Gamma_{\gamma}^{1 / 2}}{M_{R}^{2}-s-\mathrm{i} \sqrt{s} \Gamma_{t o t}}\left\{\begin{array}{l}
A_{l \pm}\left(M_{R}\right) \\
B_{l \pm}\left(M_{R}\right)
\end{array}\right\},
$$

where

$$
\Gamma_{\gamma}=\Gamma_{0}\left(\frac{k}{k_{0}}\right)^{j_{1}}\left(\frac{k_{0}^{2}+X^{2}}{k^{2}+X^{2}}\right)^{j_{2}}
$$

and

$$
\begin{aligned}
A_{l \pm}\left(M_{R}\right) & =\mp \alpha C_{N \pi} A_{1 / 2} \\
B_{l \pm}\left(M_{R}\right) & = \pm 4 \alpha[(2 J-1)(2 J+3)]^{-1 / 2} C_{N \pi} A_{3 / 2} \\
\alpha & \equiv\left[\frac{1}{\pi} \frac{k_{0}}{q_{0}} \frac{1}{2 J+1} \frac{M_{N}}{M_{R}} \frac{\Gamma_{\pi}\left(M_{R}\right)}{\Gamma_{0}^{2}}\right]^{1 / 2} .
\end{aligned}
$$

Here $k$ and $q$ are the photon and pion 3-momentum in the cms for a given cms energy $\sqrt{s} . k_{0}$ and $q_{0}$ are taken at the pole of the resonance. $\Gamma_{\pi}$ denotes the one-pion decay width and $\Gamma_{0}$ the total vacuum width at the pole of the resonance. $C_{N \pi}$ is the Clebsch-Gordan coefficient for the coupling of the isospins of the pion and the nucleon to the isospin of the resonance. The helicity amplitudes $A_{1 / 2}$ and $A_{3 / 2}$ are taken from [22]. For the parameter $X$ we use

$$
X=0.3 \mathrm{GeV}
$$

for all resonances. The parameters $j_{1}$ and $j_{2}$ are taken from [20], except for the $N(1535)$. In table 1 all parameters are listed for the resonances that are important for photoabsorption. The resonance cross section corresponding 


\begin{tabular}{|c|l|l|r|r|r|r|}
\hline \multirow{2}{*}{ resonance } & \multirow{2}{*}{$j_{1}$} & \multirow{2}{*}{$j_{2}$} & \multicolumn{2}{|c|}{$A_{1 / 2}\left[\mathrm{GeV}^{-1 / 2}\right]$} & \multicolumn{2}{|c|}{$A_{3 / 2}\left[\mathrm{GeV}^{-1 / 2}\right]$} \\
\cline { 4 - 7 } & & & $\mathrm{p}$ & $\mathrm{n}$ & $\mathrm{p}$ & $\mathrm{n}$ \\
\hline$P_{33}(1232)$ & 2 & 1 & -0.141 & -0.141 & -0.260 & -0.260 \\
\hline$D_{13}(1520)$ & 2 & 1 & -0.022 & -0.062 & 0.163 & -0.137 \\
\hline$S_{11}(1535)$ & 1 & 0 & 0.125 & -0.100 & 0 & 0 \\
\hline$F_{15}(1680)$ & 4 & 2 & -0.014 & 0.027 & 0.135 & -0.035 \\
\hline
\end{tabular}

Table 1: Resonance parameters for photoabsorption from [22, 20, 23].

to equation (6) is:

$$
\sigma_{\gamma N \rightarrow R \rightarrow N \pi}=\left(\frac{k_{0}}{k}\right)^{2} \frac{s \Gamma_{\gamma} \Gamma_{R \rightarrow N \pi}}{\left(s-M_{R}^{2}\right)^{2}+s \Gamma_{\text {tot }}^{2}} \frac{2 M_{N}}{M_{R} \Gamma_{0}}\left(\left|A_{1 / 2}\right|^{2}+\left|A_{3 / 2}\right|^{2}\right) .
$$

The parameters of the $N(1535)$ were fitted to experimental eta photoproduction data [23] that will be described in section 3.2. The helicity amplitudes extracted from pion photoproduction [22] are about a factor of 2 smaller than those in [23]. However, for the calculation of pion photoproduction this is not important since the contribution of the $N(1535)$ is anyway small.

In figure 1 we show our decomposition of the total pion photoproduction cross sections on the nucleon for all isospin channels. The total cross section is obtained by summing coherently over the individual resonance amplitudes as well as a background amplitude. The curve labeled 'background' represents the cross section that is obtained after subtracting all resonance contributions (cf. eq. (6)) from the partial-wave amplitudes [21]. The smooth background indicates that our procedure is reasonable.

An incoherent addition of all contributions, as performed by Kondratyuk et al. [8], differs significantly from the coherent one, especially in the $\Delta$-region for the $\pi^{+}$-channel. A coherent summation of the resonance and background contributions is thus mandatory if one wants to investigate possible modifications of the resonance contributions in nuclei.

Since there are no experimental data for the $\pi^{0}$-cross section on the neutron this channel suffers from the uncertainties in the partial-wave analysis from [21]. However, since the non-resonant background in this channel is at least in the region of the $\Delta$-resonance - expected to be small [19] the cross section consists mainly of resonance contributions that are fixed by measurements of the other channels and isospin symmetry. The uncertainties of the 
helicity amplitudes of the $\Delta$-resonance are only of the order of a few percent [22]. The used cross section is also in line with an extraction from the deuteron cross section [24]. Therefore we expect that our parameterizations of the one-pion photoproduction cross sections are reasonable.

\subsection{Etaproduction}

The cross section for eta photoproduction is parameterized under the assumption that the only production mechanism is that through the excitation of an intermediate $N(1535)$-resonance [23]. Then we get analogously to equation (11) the cross section within a Breit-Wigner approximation:

$$
\sigma_{\gamma N \rightarrow N \eta}=\left(\frac{k_{0}}{k}\right)^{2} \frac{s \Gamma_{\gamma} \Gamma_{N(1535) \rightarrow N \eta}}{\left(s-M_{1535}^{2}\right)^{2}+s \Gamma_{t o t}^{2}} \frac{2 M_{N}}{M_{1535} \Gamma_{0}}\left|A_{1 / 2}\right|^{2},
$$

where $\Gamma_{\gamma}$ has been defined in equation (17). The total width $\Gamma_{t o t}$ is given by:

$$
\Gamma_{t o t}=\Gamma_{\pi}+\Gamma_{\eta}+\Gamma_{\pi \pi}
$$

with

$$
\begin{aligned}
\Gamma_{\pi} & =\frac{q_{\pi}}{q_{R, \pi}} b_{\pi} \Gamma_{0} \frac{q_{R, \pi}^{2}+c_{\pi}^{2}}{q_{\pi}^{2}+c_{\pi}^{2}} \\
\Gamma_{\eta} & =\frac{q_{\eta}}{q_{R, \eta}} b_{\eta} \Gamma_{0} \frac{q_{R, \eta}^{2}+c_{\eta}^{2}}{q_{\eta}^{2}+c_{\eta}^{2}} \\
\Gamma_{\pi \pi} & =b_{\pi \pi} \Gamma_{0} \Theta\left(\sqrt{s}-M_{N}-2 m_{\pi}\right) .
\end{aligned}
$$

The position of the resonance pole $M_{1535}$ and the total width $\Gamma_{0}$ with the branching ratios $b_{\pi}, b_{\eta}$ and $b_{\pi \pi}$ as well as the helicity amplitude $A_{1 / 2}$ are adopted from [23]. The fit to the experimental data was improved by multiplying form factors with parameters $c_{\pi}$ and $c_{\eta}$ to the partial widths. With

$$
c_{\pi}^{2}=c_{\eta}^{2}=0.25 \mathrm{GeV}^{2}
$$

one obtains the solid curve shown in figure 2. For photon energies larger than $800 \mathrm{MeV}$ only electroproduction data with a low virtual photon momentum transfer of $q^{2}=-0.056 \mathrm{GeV}^{2}$ are available 25]. Following [26] where it is argued that these data can be regarded as photoproduction data within the 
errorbars, we will also use a different fit including these data points (dashed curve in figure 2) for comparison. This will only affect the electromagnetic properties of the $N(1535)$-resonance, but not the hadronic ones.

For the neutron cross section we use the proton cross section scaled by a factor 0.64 according to [27].

\subsection{Two-pion production}

In figure 3 we compare the contributions of the $N(1520)$ and the $N(1680)$ with the measured two-pion photoproduction cross sections on the proton for the three isospin channels. The resonance contributions are given by the Breit-Wigner formula in equation (11) with $\Gamma_{R \rightarrow N \pi}$ replaced by $\Gamma_{R \rightarrow N \pi \pi}$. It is obvious that these contributions explain neither the size nor the structure of the cross section for any channel.

There are calculations of the two-pion photoproduction cross section by L. Y. Murphy et al. 29] and J. A. Gomez Tejedor et al. [30] within the framework of a phenomenological pion nucleon field theory. In both calculations the number of diagrams giving important contributions is rather large with, unfortunately, only little agreement in the assignment of the most important ones. Therefore one can expect insight into the underlying processes only by the experimental measurement of massdifferential cross sections. Up to then we treat the difference between the experimental cross section and the BreitWigner type resonance contributions as background, where the momenta of the outgoing particles are distributed according to three-body phase space. Therefore the only medium modification is the possible Pauli blocking of the outgoing nucleon. This approach is somewhat unsatisfactory since the 'background' is probably dominated more by intermediate excitations of baryonic and mesonic resonances than by contributions coming from diagrams involving pions and nucleons only. For photon energies that exceed the measured energy range we assume the cross sections to be constant.

Because there are no recent data available for $2 \pi$ production on the neutron we obtain these from the difference between the total photoabsorption cross section and the one-pion photoproduction cross section. We use an ansatz for the background contribution:

$$
\sigma_{\gamma n \rightarrow N \pi \pi}^{b g}=\frac{a_{1} x+a_{2} x^{2}}{1+a_{3} x+a_{4} x^{2}} \mu \mathrm{b}
$$




$$
x=\frac{\sqrt{s}-M_{N}-2 m_{\pi}}{\mathrm{GeV}}
$$

and obtain for the fit parameters:

$$
a_{1}=249.7 \quad a_{2}=314.1 \quad a_{3}=-0.3537 \quad a_{4}=4.604 \quad .
$$

The decomposition into the different isospin channels is done by using the maximum values of the two-pion cross sections on the proton. This gives independent of photon energy:

$$
\sigma_{\gamma n \rightarrow n \pi^{+} \pi^{-}}: \sigma_{\gamma n \rightarrow p \pi^{-} \pi^{0}}: \sigma_{\gamma n \rightarrow n \pi^{0} \pi^{0}}=75: 55: 12
$$

\subsection{Implementation into the BUU model}

In the BUU model nucleon resonances are explicitly propagated in order to account for multi-step processes. Therefore we replace the coherent sum for the one-pion photoproduction cross section by a properly scaled incoherent cross section. This gives an effective cross section for the photoproduction of a resonance $\mathrm{R}$ :

$$
\sigma_{\gamma N \rightarrow R}^{e f f}=\int \frac{\frac{d \sigma_{\gamma N \rightarrow R \rightarrow N \pi}}{d \Omega}}{\frac{d \sigma_{\gamma N \rightarrow N \pi}^{b g}}{d \Omega}+\sum_{R^{\prime}} \frac{d \sigma_{\gamma N \rightarrow R^{\prime} \rightarrow N \pi}}{d \Omega}} \frac{d \sigma_{\gamma N \rightarrow N \pi}}{d \Omega} d \Omega+\sigma_{\gamma N \rightarrow R \rightarrow X(X \neq N \pi)}
$$

where $\sigma_{\gamma N \rightarrow R \rightarrow N \pi}$ and $\sigma_{\gamma N \rightarrow N \pi}^{b g}$ denote the cross sections without interferences. The sum $\sum_{R^{\prime}}$ runs over all contributing resonances. Analogously the cross section for the direct production of a $N \pi$-pair is given by:

$$
\frac{d \sigma_{\gamma N \rightarrow N \pi}^{b g, e f f}}{d \Omega}=\frac{\frac{d \sigma_{\gamma N \rightarrow N \pi}^{b g}}{d \Omega}}{\frac{d \sigma_{\gamma N \rightarrow N \pi}^{b g}}{d \Omega}+\sum_{R} \frac{d \sigma_{\gamma N \rightarrow R \rightarrow N \pi}}{d \Omega}} \frac{d \sigma_{\gamma N \rightarrow N \pi}}{d \Omega} .
$$

This procedure guarantees that the sum of these cross sections is equal to the cross section obtained by coherent addition of all contributions.

From figure 1 one sees that for the channels with charged pions the size of the interference terms of resonance and background contributions amounts to at most about $20 \%$ of the total one-pion cross sections. Since for all resonances the width for spontaneous decay is much larger than the collision width (see [1]) the uncertainties induced by our decomposition of the cross section into a sum of incoherent contributions are negligible. 
The medium modifications for the elementary cross sections are described in [1]. The vacuum width appearing in the resonance propagator in equation (6) is replaced by the in-medium width as calculated in [1]. The collision width gives a contribution to the absorption cross section of the form of equation (11) with $\Gamma_{R \text {,coll }}$ instead of $\Gamma_{R \rightarrow N \pi}$. For the $\Delta$-resonance the difference between nucleon and $\Delta$-potential (equation (5)) causes a real part of the self energy $\Pi$ to be used in the resonance propagator:

$$
\operatorname{Re} \Pi=2 E_{\Delta}\left(U_{N}-U_{\Delta}\right)
$$

We also take into account that nucleon final states can be Pauli blocked. The decay of the resonances is, for simplicity, always assumed to be isotropic in the resonance rest frame.

\section{Results}

\subsection{Pion photoproduction}

In figure 1 we show the time evolution of the photon nucleus reaction for different nuclei and photon energies of 300 and $750 \mathrm{MeV}$. The duration of the reaction is about $20 \mathrm{fm} / \mathrm{c}$ and more or less independent of the photon energy. For both photon energies the $\Delta$-resonance plays an important role, but its time-development is quite different. Whereas at $E_{\gamma}=300 \mathrm{MeV}$ the main $\gamma$ absorption goes through the $\Delta$ which subsequently decays, at $750 \mathrm{MeV}$ only a small number of $\Delta$ 's is directly excited by the photon. Most of the $\Delta$ 's are populated by collisions of nucleons with pions from the absorption process $\gamma N \rightarrow N \pi \pi$. From the decay time of the $\Delta$-contribution, being much larger than the lifetime of a single $\Delta$, one can see that the reaction is indeed a multi-step process dominated by continous excitation and decay of $\Delta$-resonances. The excitation propagates alternately as $\Delta$ or pion through the nuclear medium.

The total $\pi^{0}$ cross section on ${ }^{12} \mathrm{C}$ is shown in figure 5. The curve labeled 'without medium modifications' results when applying only Fermi motion and Pauli blocking and neglecting the difference between nucleon- and $\Delta$ potential and the in-medium widths of the resonances. The difference to the full calculation is basically caused by the $\Delta$-potential. In the region of the $\Delta$-resonance the elementary absorption cross section is reduced and 
shifted to higher energies [1]. For larger photon energies the $\Delta$-potential leads to a slight enhancement of the pion production due to its effect on the pion-nucleon interaction. Compared to the experimental data from [15] our calculation overestimates the cross section in the region of the $\Delta$-resonance by about 30\%. In recent experiments in Mainz 31] only pions within a 'missing energy' window were detected. The cut on the 'missing energy' $E_{\text {mis }}$ led to a preferred detection of quasi-free produced pions which had no final state interaction. In the pole region of the $\Delta$-resonance we again overestimate the cross section by about the same factors as the total cross section and at the high energy tail we underestimate it but here the cross section is too small to draw any firm conclusions.

In figure 6 we show our results on ${ }^{40} \mathrm{Ca}$ and ${ }^{208} \mathrm{~Pb}$. Qualitatively the curves look similar to the ones for ${ }^{12} \mathrm{C}$. The cross section on ${ }^{208} \mathrm{~Pb}$ shows somewhat better agreement with the experimental data than in the case of ${ }^{12} \mathrm{C}$, but we again fail to explain the broad structure in the $\Delta$-region.

The photoproduction of charged pions in nuclei was measured only up to photon energies of $400 \mathrm{MeV}$ [32]. Similar to the $\pi^{0}$ production we overestimate these cross sections by about $25 \%$.

The discrepancy in the $\Delta$-region might be due to a further reduction of the cross section for $\gamma N \rightarrow N \pi$ in the nuclear medium. A better description of the total photoabsorption cross section then certainly requires the inclusion of two- and three-body absorption mechanisms for the photon. This may also lead to a better description of the observed structure of the cross sections. However, one has to note that only a part of all produced pions actually survives the final state absorption on their way out of the nucleus. Thus, one needs a very reliable description of pion-absorption in order to become sensitive to modifications of the elementary photon-nucleon interaction.

The shape of the energy and angular differential cross sections is nearly independent of the mass of the nucleus. Therefore we limit ourselves here to the discussion of the results for ${ }^{40} \mathrm{Ca}$. In figure 7 angular differential cross sections for the production of $\pi^{0}$ 's are shown for different photon energies. From the difference between the curves labeled 'Fermi motion alone' and ' + isotropic resonance' one sees that for photon energies less than 500 $\mathrm{MeV}$, where the p-state interaction dominates, the isotropic resonance decay, assumed in the BUU model, leads to an incorrect implementation of the elementary angular differential cross sections. However, the final state interaction of the pions with the nuclear medium leads in general to a higher 
isotropy of the cross section so that the final result is not affected by this deficiency. For small photon energies the cross section at forward angles is additionally reduced since a pion emitted at forward angles corresponds to a low momentum nucleon which has a high probability to be Pauli blocked. For higher photon energies this becomes more and more unimportant and the cross section is shifted to forward angles because of kinematical reasons.

Momentum differential cross sections for the same energy ranges of the photon are given in figure 8. Here the influence of the isotropic resonance decay is less important. The structure in the elementary cross section between photon energies of 450 and $650 \mathrm{MeV}$ coming from the one-pion and two-pion contribution is already washed out by Fermi motion. Independent of photon energy one observes an enhancement of the cross section at pion momenta of $200 \mathrm{MeV}$ and a reduction at $400 \mathrm{MeV}$. This is due to the strong final state coupling of the pions to nucleons as discussed earlier. For smaller pion momenta the pion nucleon cross section, dominated here by the $\Delta$-resonance, gets smaller. Therefore pions with momenta around $200 \mathrm{MeV}$ have a higher probability to escape from the nucleus.

In figures 8 and 8 it is noticeable that the $\pi^{0}$ cross section compared to the elementary cross section is in the $\Delta$-region $\left(E_{\gamma}=300-350 \mathrm{MeV}\right)$ much smaller than for higher energies. This is due to charge exchange reactions by primary produced charged pions, whose relative contribution to the elementary cross section increases with increasing photon energy.

An experimental measurement of pion photoproduction in nuclei in the energy range from $400 \mathrm{MeV}$ to $1 \mathrm{GeV}$ would be very helpful because with increasing photon energy multi-body absorption mechanisms are expected to become smaller. Then a comparison between theory and experiment would allow cleaner conclusions about medium modifications of the elementary photon-nucleon interaction.

Figure 9 shows the inclusive two-pion photoproduction cross sections on ${ }^{12} \mathrm{C}$ and ${ }^{40} \mathrm{Ca}$. Since there are yet no experimental data available we compare our results with the calculations of J. A. Gomez Tejedor et al. [33] done in the framework of a $\Delta$-hole model. The difference to our calculation is due to the fact that in our calculation there is only a small medium modification of the elementary $\gamma N \rightarrow N \pi \pi$ cross section. In [33] this cross section is calculated microscopically using medium modifications for the propagators and potentials for the phasespace integrations that cause a strong enhancement of the elementary cross section in the nuclear medium. The differences 
between the two $\pi^{+} \pi^{-}$curves are so large that they should be experimentally observable. Such a measurement would also be very helpful with respect to the disappearance of the $D_{13}$ in the total photoabsorption cross section [1].

\subsection{Etaproduction}

In section 3.2 we have parameterized the etaproduction on the free nucleon under the assumption that the only production mechanism is via an intermediate $N(1535)$-resonance. In nuclei we also have to take into account eta production by final state interactions of pions that were primary produced.

In figure 10 we compare the calculated total etaproduction cross section on ${ }^{12} \mathrm{C}$, ${ }^{40} \mathrm{Ca}$ and ${ }^{208} \mathrm{~Pb}$ with experimental data from [34. The contributions coming from secondary processes are almost negligible. For large photon energies our calculation depends on the choice of the elementary $\gamma N \rightarrow N \eta$ cross section for photon energies larger than $800 \mathrm{MeV}$ because of the Fermi motion of the nucleons. An extrapolation of this cross section according to low momentum transfer electroproduction data as discussed in chapter 3.2 (dashed curve in figure 2) reduces the total cross section at $800 \mathrm{MeV}$ by about $15 \%$ and gives a better description of the experimental data. On ${ }^{12} \mathrm{C}$ there is only good agreement with the experiment at low photon energies. For higher energies we overestimate the cross section by about $20 \%$. On ${ }^{40} \mathrm{Ca}$ and ${ }^{208} \mathrm{~Pb}$ the agreement with the experiment is very good.

Angular differential cross sections on ${ }^{40} \mathrm{Ca}$ are shown together with the experimental data in figure 11 for different photon energies. For comparison we also show the cross sections on a free nucleon and the cross sections that result when applying Fermi motion alone and neglecting any medium effects on the N(1535)-resonance. The influence of the final state interaction on the shape of the angular differential cross sections is small. Compared to the data the calculated cross sections are slightly shifted to smaller angles. The same holds for ${ }^{12} \mathrm{C}$ and ${ }^{208} \mathrm{~Pb}$.

A corresponding discrepancy can be seen in energy differential cross sections given in figure 12. Here the discrepancy is larger; the calculated cross sections are shifted to larger eta energies since etas emitted at forward angles have larger energies.

Possible reasons for the discrepancies in the shape of the observed differential cross sections are an eta potential and a modification of the cross

section for eta-nucleon scattering. In order to check these possibilities we 
have first used an eta potential as given by Lee et al. [35]. This potential is attractive for eta energies below $100 \mathrm{MeV}\left(-30 \mathrm{MeV}\right.$ for $T_{\eta}=0$ at $\left.\rho_{0}\right)$ and slightly repulsive for larger energies. In our calculation the potential enters the phase space integration for the eta production as well as the equations of motions for the propagation of the eta through the nucleus. In figure 13 the resulting differential cross sections on ${ }^{40} \mathrm{Ca}$ for a photon energy of 780 $\mathrm{MeV}$ are shown with the previous obtained cross section. For simplicity we now neglect contributions from primary produced pions or resonances other than N(1535) since they are anyway small. From figure 13 one sees that the effect of the potential is quite small.

Since only a small fraction of the etas produced inside the nucleus survives the propagation through the nucleus (about $1 / 3$ for ${ }^{40} \mathrm{Ca}, 1 / 6$ for ${ }^{208} \mathrm{~Pb}$ ) there is a strong dependence of the eta production cross sections on modifications of the eta absorption processes. Indeed we find that we are able to reproduce the experimental data by using a constant inelastic $\eta N$-cross section, $\sigma_{i n .}^{\eta}=$ $30 \mathrm{mb}$, and a constant elastic cross section, $\sigma_{e l}^{\eta}=20 \mathrm{mb}$. The resulting cross sections are labeled 'modified $\eta$-rescattering' in figure 13. One should note that energy and angular differential cross sections are then reproduced simultaneously.

\section{$5 \quad$ Summary and outlook}

We have presented a calculation of the photoproduction of pions and etas within the framework of a semi-classical BUU transport model for photon energies from 300 to $900 \mathrm{MeV}$. Starting from a realistic parameterization of the free photon-nucleon cross sections we have applied the medium modifications Fermi motion, Pauli blocking and collision broadening for the involved nucleon resonances.

In the $\Delta$-region we are able to reproduce the size of the observed pion production in nuclei reasonably well although our calculated cross sections show too much structure in the resonance region. This might be due to the importance of multi-body absorption mechanisms. Our results for two-pion production differ by about a factor of 2 from calculations within the $\Delta$-hole model. This difference can only clarified by a measurement.

The agreement of the calculated total eta production cross section with the experiment is good. The angular and energy differential cross sections 
show a systematic deviation from the experiment for all nuclei and photon energies. This can be corrected by a phenomenological adjustment of the cross section for $\eta N$-scattering but it might as well be due to an effect of the eta or nucleon potential which is not accessible in our semi-classical calculation or to shortcomings in our description of $\eta$-rescattering.

An experimental measurement of exclusive cross sections, i. e. one-pion production, two-pion production, nucleon knock-out etc., is imperative for a better understanding of the photon-nucleus reaction and an explanation of the disappearance of the $D_{13}$-resonance in the total photonuclear absorption cross section. Such measurements would help to separate possible in-medium modifications of the elementary $(\gamma, N)$ reactions from final state interactions of the produced particles.

Nucleon knock-out reactions are of particular interest with respect to multi-body absorption mechanisms. A comparison between our model and experiments performed in Mainz [38] will be available soon.

\section{References}

[1] M. Effenberger et al., University of Giessen preprint, 1996, nuclth/9607005.

[2] T. Frommhold et al., Phys. Lett. B295 (1992).

[3] N. Bianchi et al., Phys. Lett. B325 (1994).

[4] M. MacCormick et al., Phys. Rev. C53 (1996) 41.

[5] T. A. Armstrong et al., Phys. Rev. D5 (1972) 1640.

[6] T. A. Armstrong et al., Nucl. Phys. B41 (1972) 445.

[7] W. M. Alberico et al., Phys. Lett. B321 (1994) 177.

[8] L. A. Kondratyuk et al., Nucl. Phys. A579 (1994) 453.

[9] N. Bianchi et al., LNF-95-053-P (1995), Phys. Rev. C in press.

[10] G. F. Bertsch et al., Phys. Rev. C29 (1984) 673. 
[11] W. Cassing et al., Phys. Rep. 188 (1990) 363.

[12] S. Teis et al., University of Giessen preprint, 1996, nucl-th/9609009, Z. Phys. $\mathbf{A}$ in press.

[13] Gy. Wolf et al., Nucl. Phys. A552 (1993) 549.

[14] A. Engel et al., Nucl. Phys. A572 (1994) 657.

[15] J. Arends et al., Nucl. Phys. A454 (1986) 579.

[16] R. C. Carrasco et al., Nucl. Phys. A541 (1992) 585.

[17] A. Hombach et al., Z. Phys. A352 (1995) 223.

[18] T. Hatsuda et al., Prog. Theor. Phys. 95 (1996) 1009.

[19] T. Ericson and W. Weise, Pions and nuclei, Clarendon Press, Oxford (1988).

[20] R. L. Walker, Phys. Rev. 182 (1969) 1729.

[21] R. A. Arndt et al., Phys. Rev. C42 (1990) 1853.

[22] Particle Data Group, Phys. Rev. D50 (1994) 1173.

[23] B. Krusche et al., Phys. Rev. Lett. 74 (1995) 3736.

[24] B. Krusche, private communication.

[25] B. Schoch, Prog. Part. Nucl. Phys. 34 (1995) 43.

[26] N. Kaiser et al., hep-ph/9607459.

[27] B. Krusche et al., Phys. Lett. B358 (1995) 40.

[28] A. Braghieri et al., Phys. Lett. 363 (1995) 46.

[29] L. Y. Murphy and J.-M. Laget, Preprint CEA/DAPNIA/SPhn 9444.

[30] J. A. Gomez Tejedor and E. Oset, Nucl. Phys. A571 (1994) 667.

[31] B. Krusche, Proc. of the Workshop on Production, Properties and Interaction of Mesons, Krakau, 1996. 
[32] J. Arends et al., Z. Phys. A305 (1982) 205.

[33] J. A. Gomez Tejedor et al., Nucl. Phys. A588 (1995) 819.

[34] M. E. Röbig-Landau et al., Phys. Lett. B373 (1996) 45.

[35] F. X. Lee et al., Nucl. Phys. A603 (1996) 345.

[36] D. Menze et al., ZAED Compilation of pion photoproduction data, Universitt Bonn, 1977.

[37] Baldini et al., Landolt-Börnstein, Band 12, Springer Verlag, Berlin, 1987.

[38] G. Cross et al., Nucl. Phys. A593 (1995) 463. 

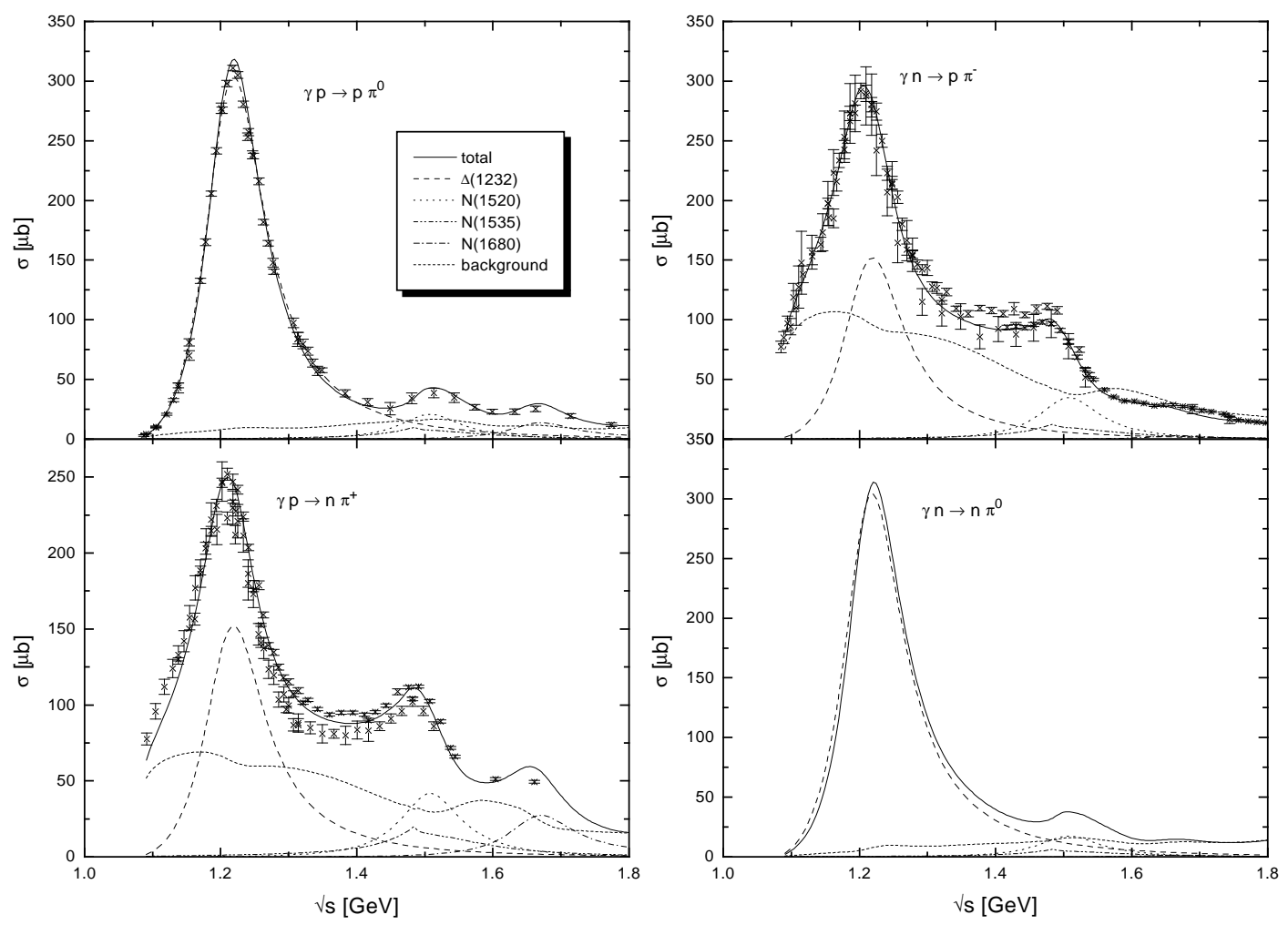

Figure 1: Resonance and background contributions for one-pion photoproduction cross sections on the nucleon. The experimental data are from [36]. 


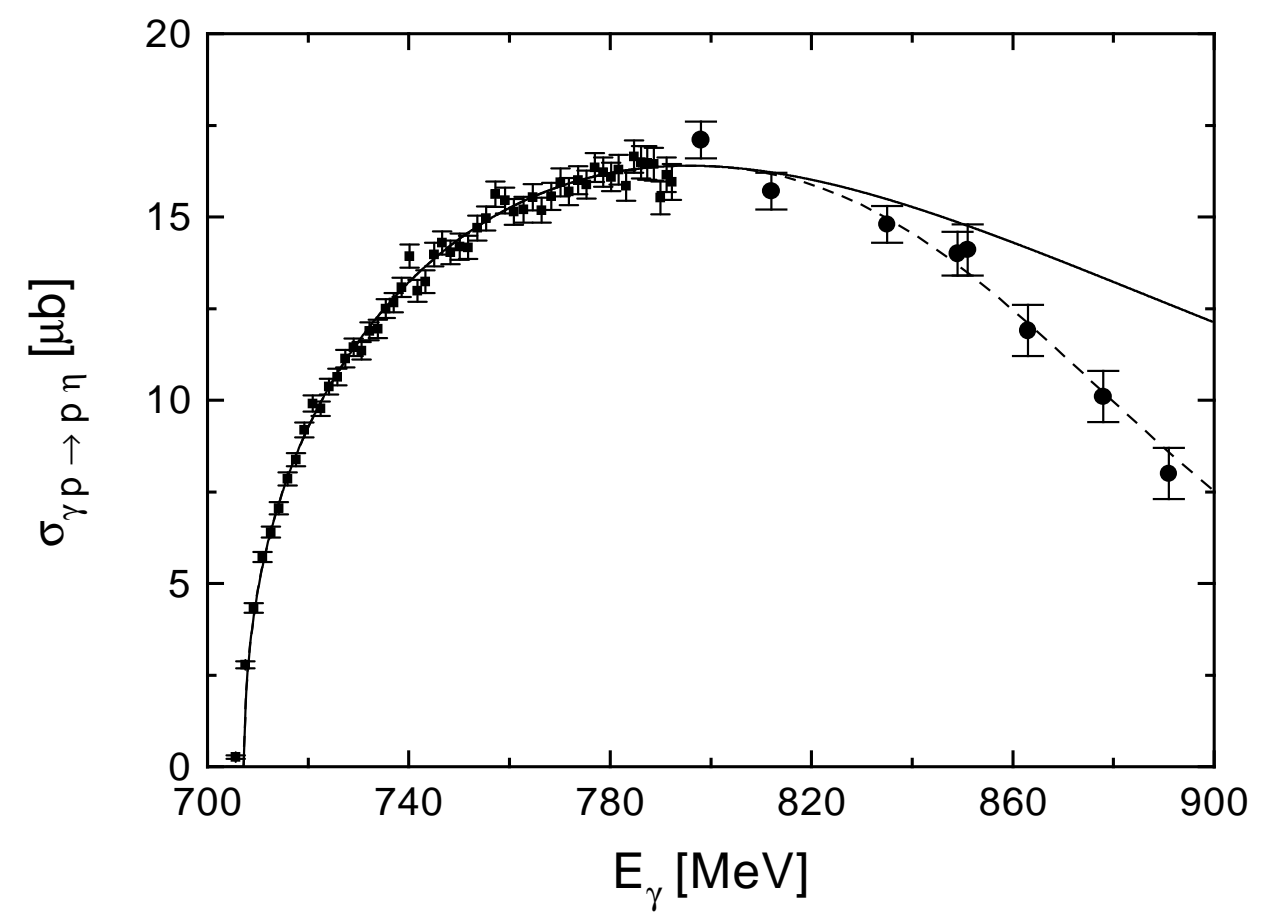

Figure 2: Cross section for eta photoproduction on the proton. The experimental data are taken from [23] (squares) and [25] (circles). Explanation see text. 

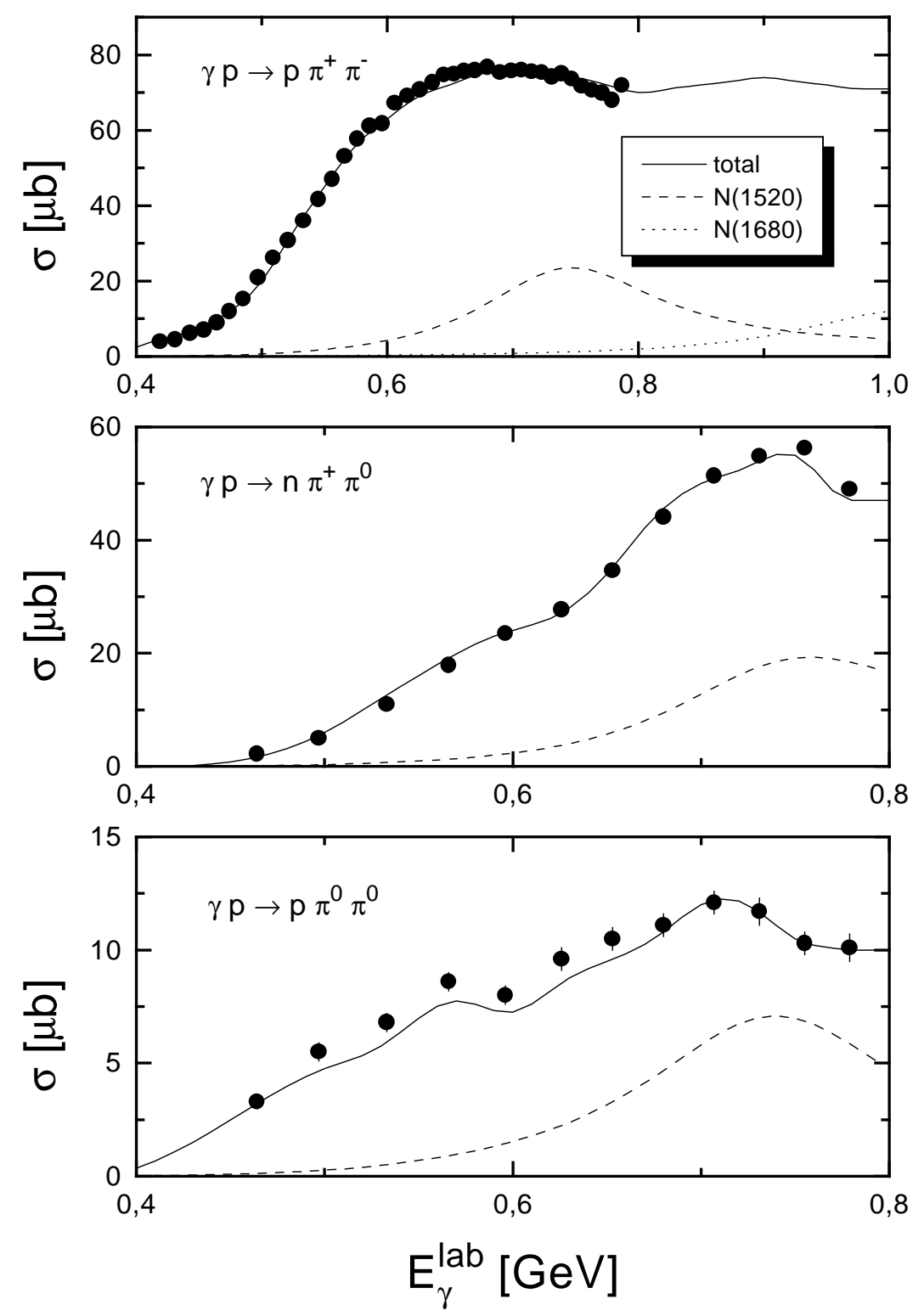

Figure 3: Cross sections for two-pion photoproduction on the proton. The experimental data are from [28]. 


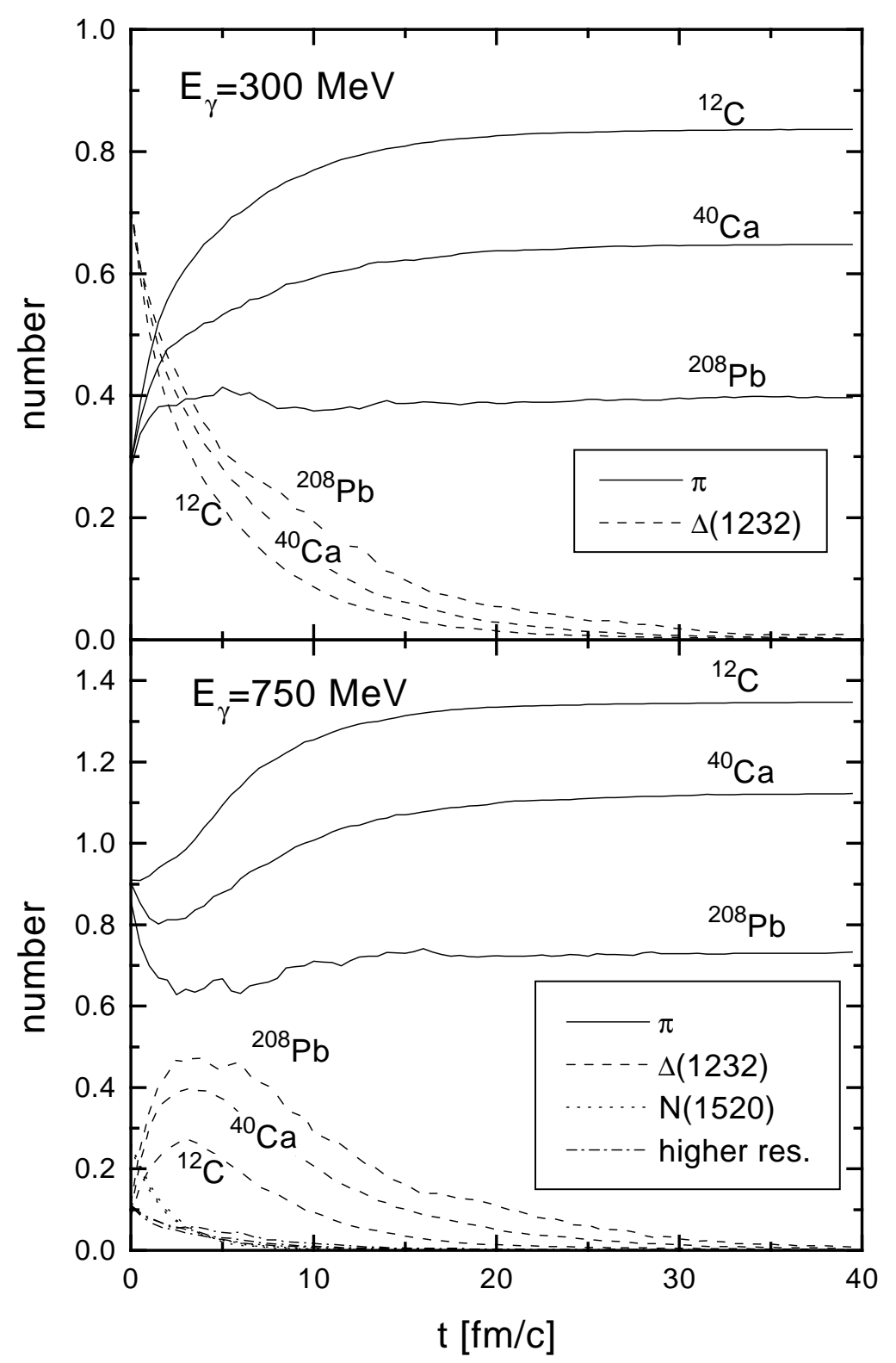

Figure 4: Time evolution of the photon-nucleus reaction. The curves represent average numbers of particles (integrated over isospin) per absorbed photon in a nucleus. 


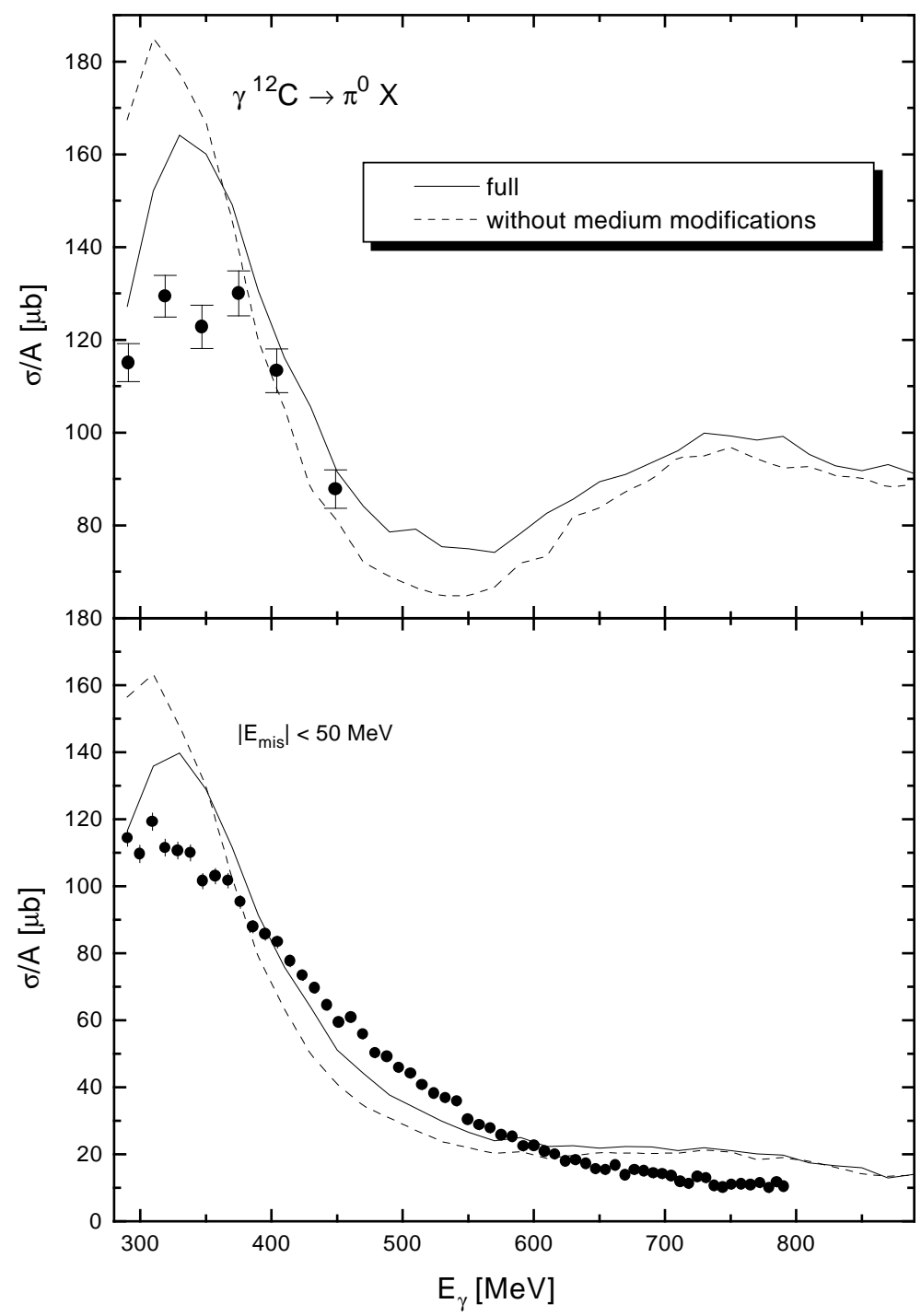

Figure 5: $\pi^{0}$ photoproduction cross sections on ${ }^{12} \mathrm{C}$. The upper figure shows the total cross section (experimental data are from [15]). The lower figure depicts the cross section with a cut on the energy of the $\pi^{0}$ (see text, experimental data from [31]). The dashed curves result when using the vacuum widths for the resonances and neglecting the difference between nucleon- and $\Delta$-potential. 


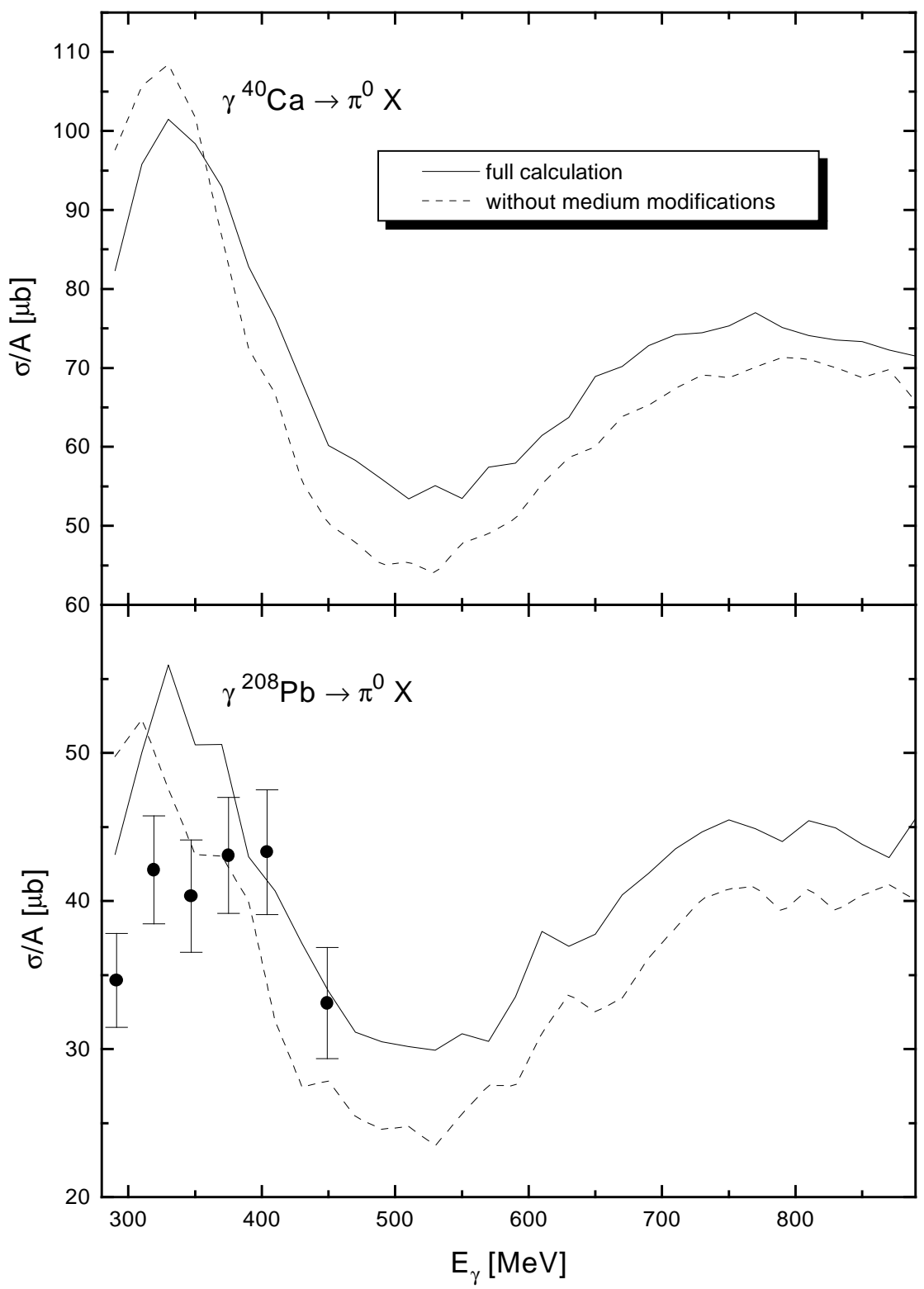

Figure 6: Total $\pi^{0}$ photoproduction cross sections on ${ }^{40} \mathrm{Ca}$ and ${ }^{208} \mathrm{~Pb}$. The experimental data are taken from [15]. The fluctuations in the calculated curves are caused by low statistics. 

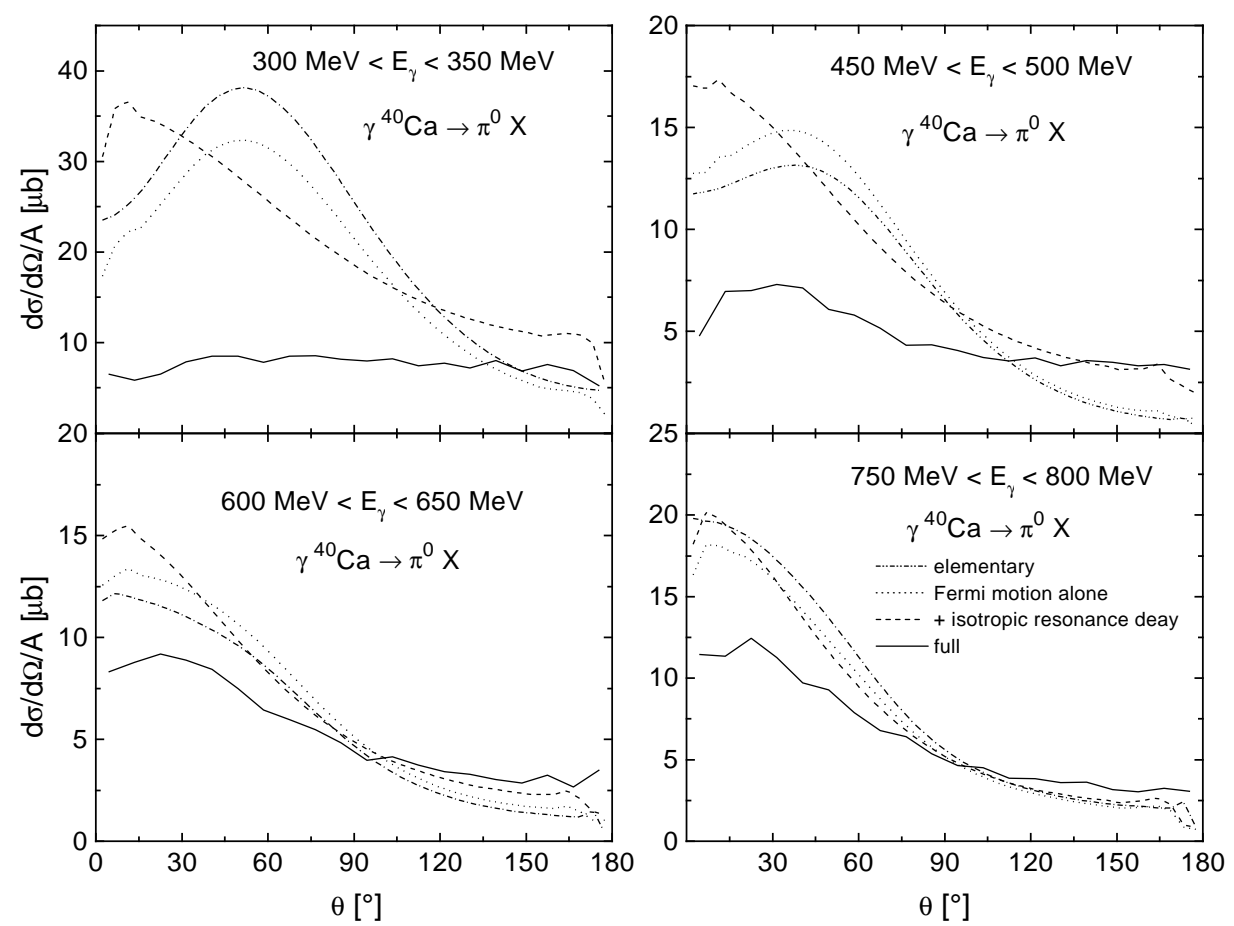

Figure 7: Angular differential $\pi^{0}$ photoproduction cross sections on ${ }^{40} \mathrm{Ca}$ for different photon energies. The dash-dotted curves show the cross sections on a free nucleon. Applying Fermi motion alone leads to the dotted curves. An isotropic resonance decay, which is assumed in the BUU calculation, gives the dashed curves (without final state interactions of the outgoing pions). The solid lines represent the full BUU calculation. 

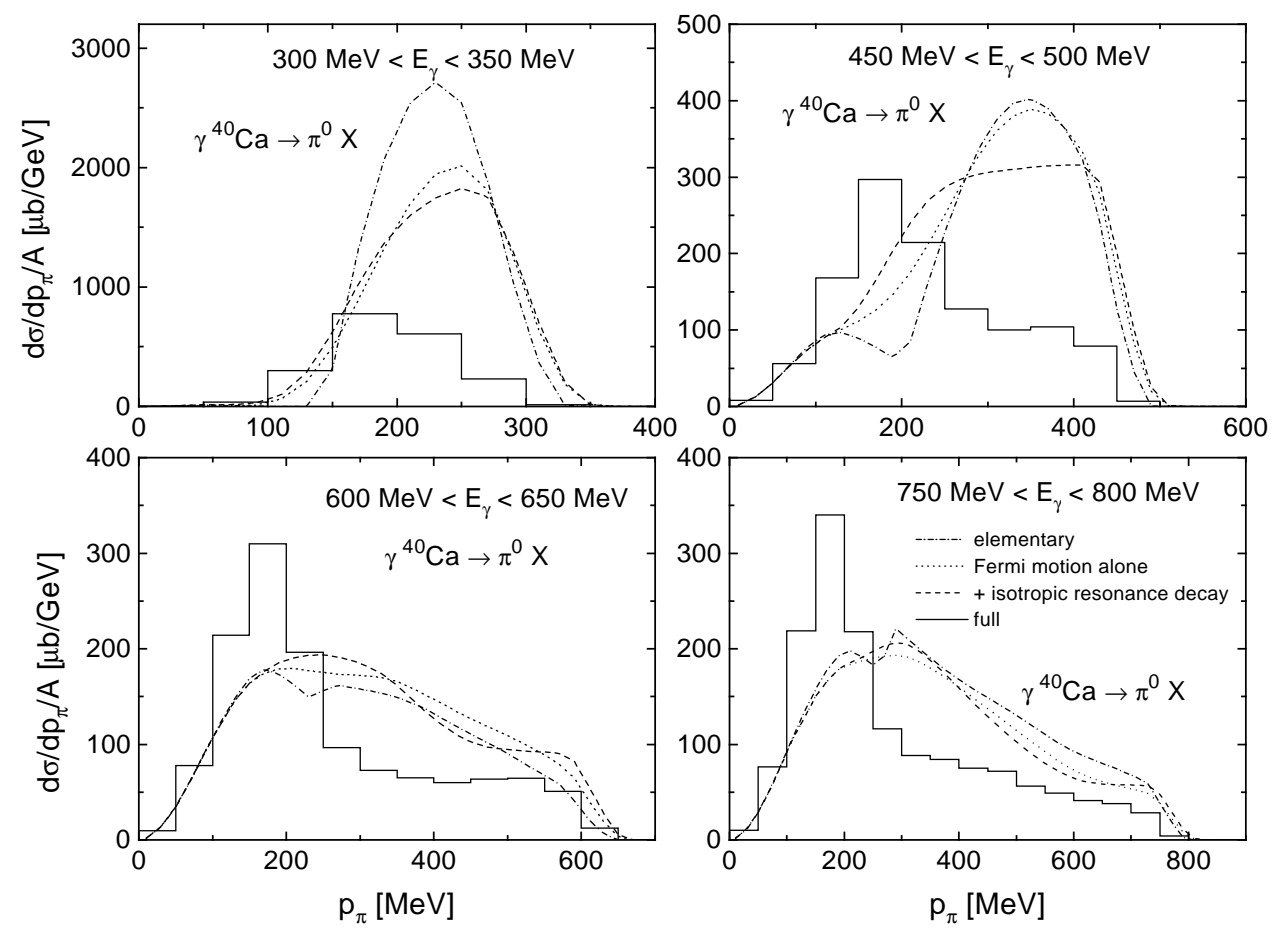

Figure 8: Momentum differential $\pi^{0}$ photoproduction cross sections on ${ }^{40} \mathrm{Ca}$ for different photon energies. Explanation see figure 7 . 


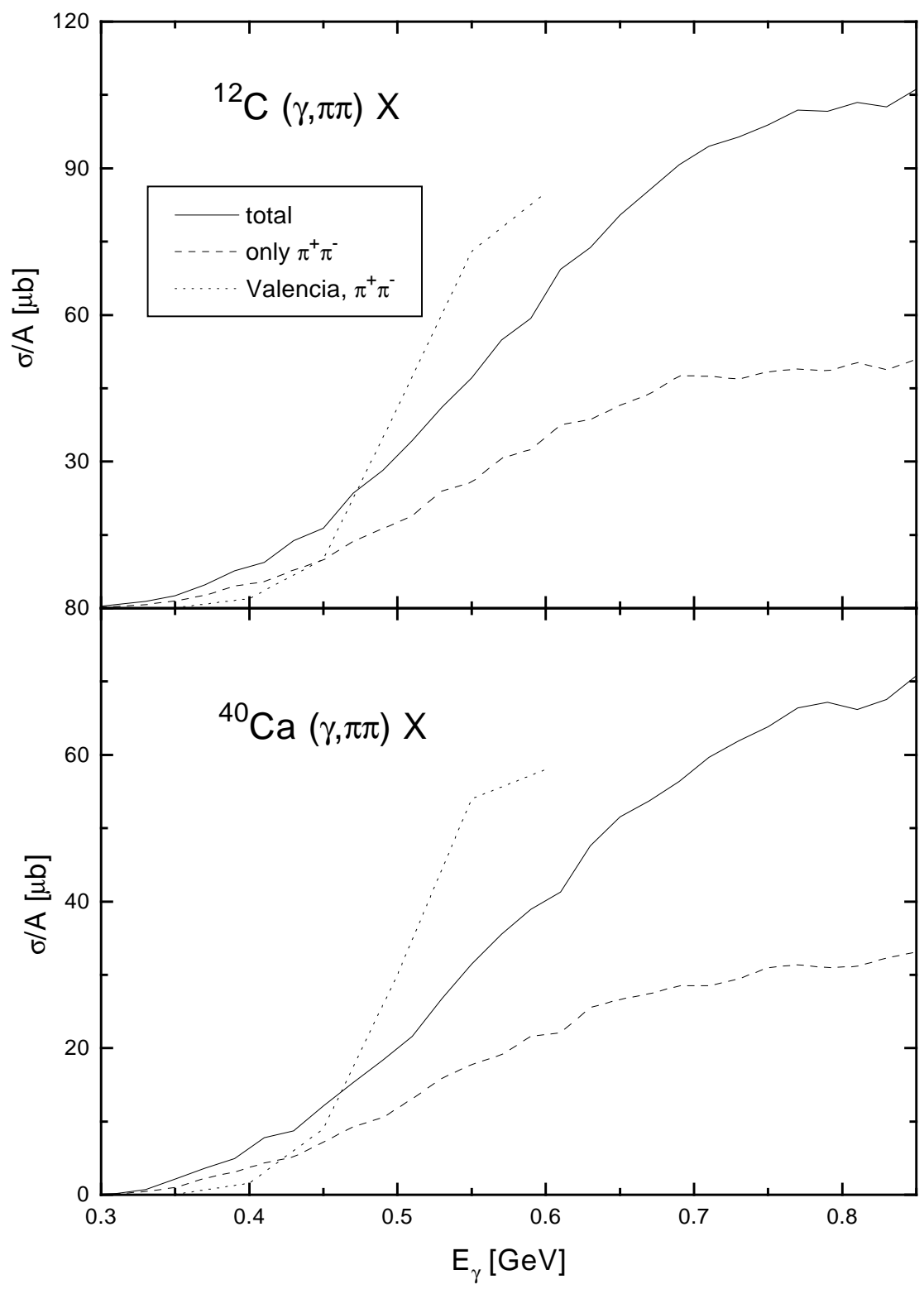

Figure 9: Total two-pion photoproduction cross section on ${ }^{12} \mathrm{C}$ and ${ }^{40} \mathrm{Ca}$. The curve labeled 'Valencia' is taken from a calculation of J. A. Gomez Tejedor et al. 33 . 


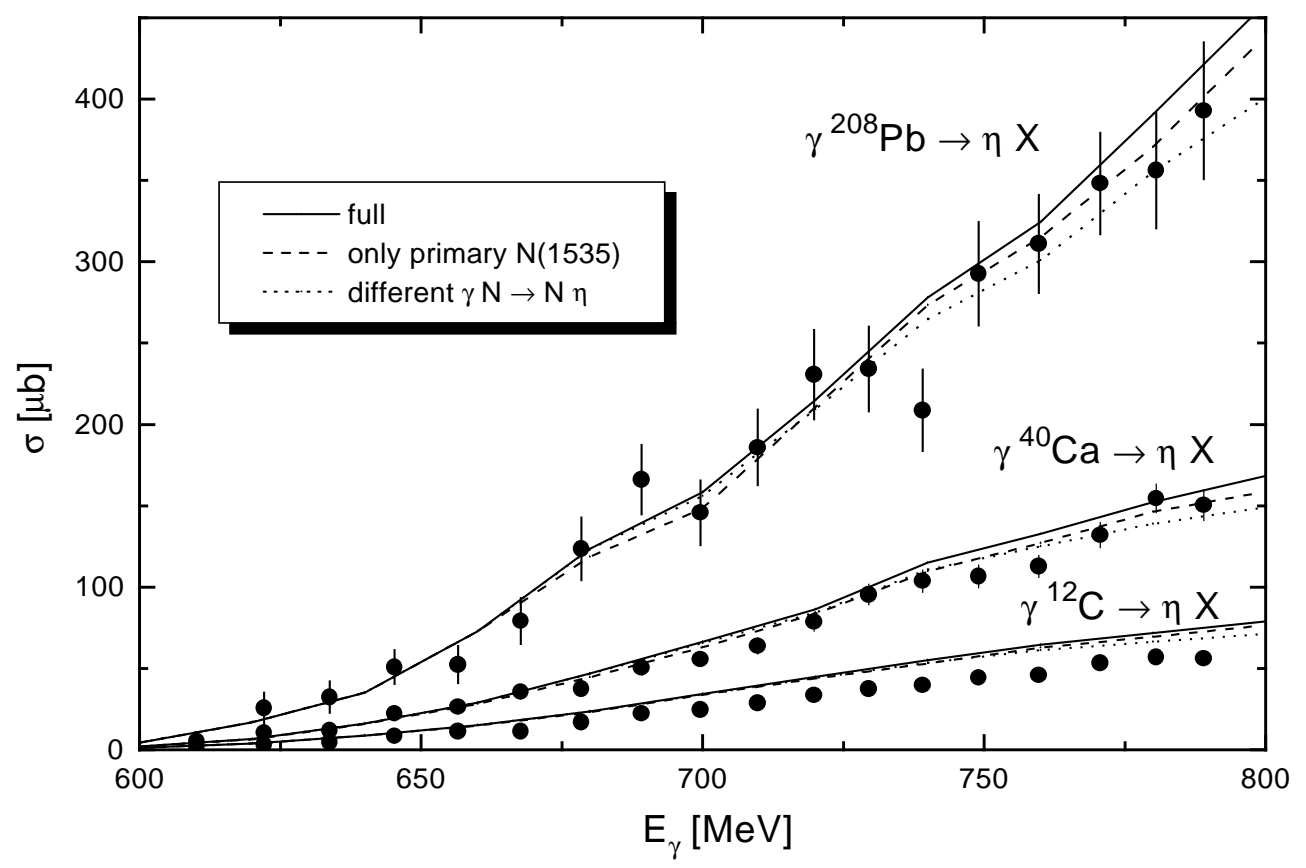

Figure 10: Total eta photoproduction cross section on ${ }^{12} \mathrm{C},{ }^{40} \mathrm{Ca}$ and ${ }^{208} \mathrm{~Pb}$. All experimental eta photoproduction data are taken from [34]. 

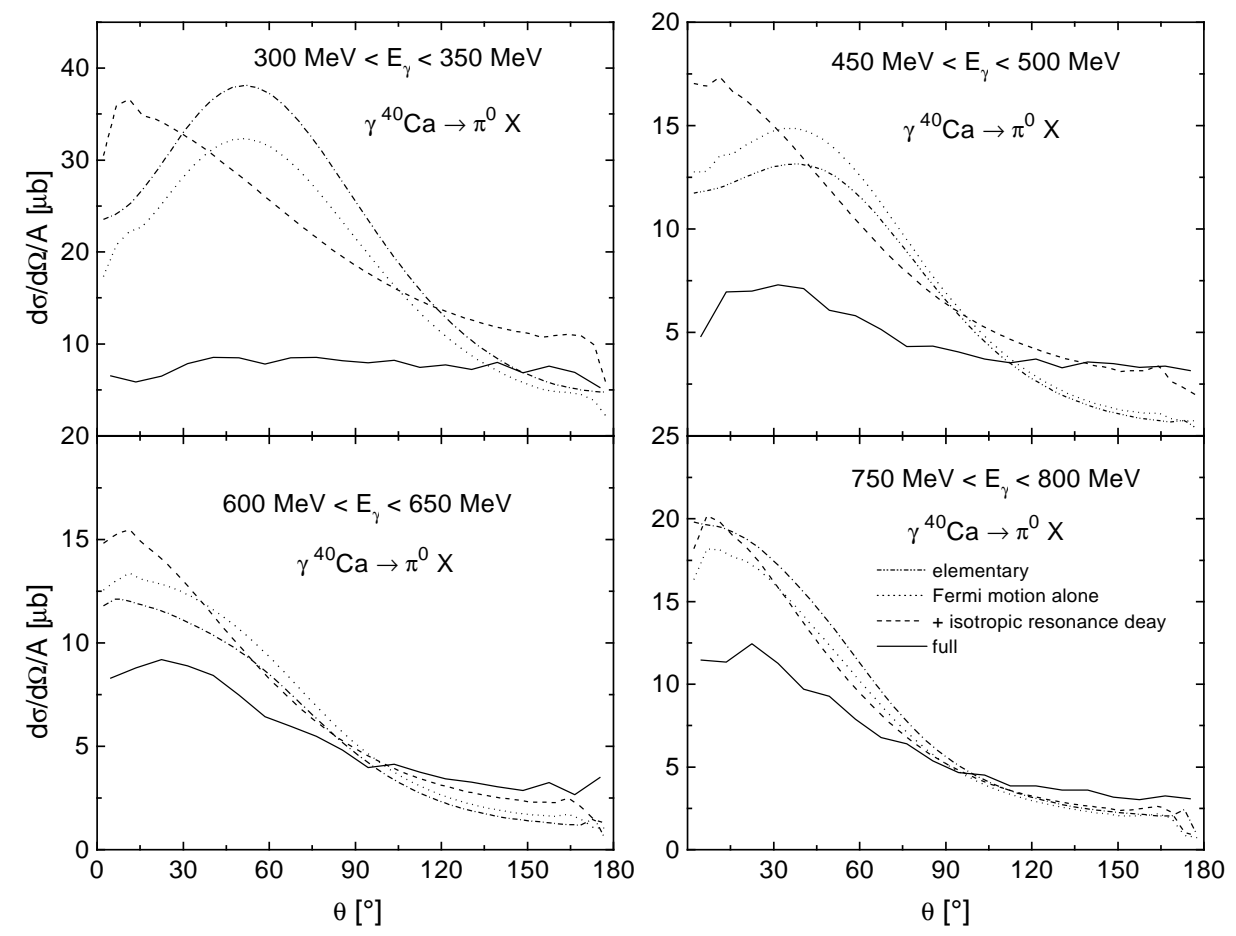

Figure 11: Angular differential eta photoproduction cross sections on ${ }^{40} \mathrm{Ca}$. The dashed curves result when applying Fermi motion alone without any final state interaction of the outgoing etas. The dotted lines represent the cross sections on a free nucleon at rest. 

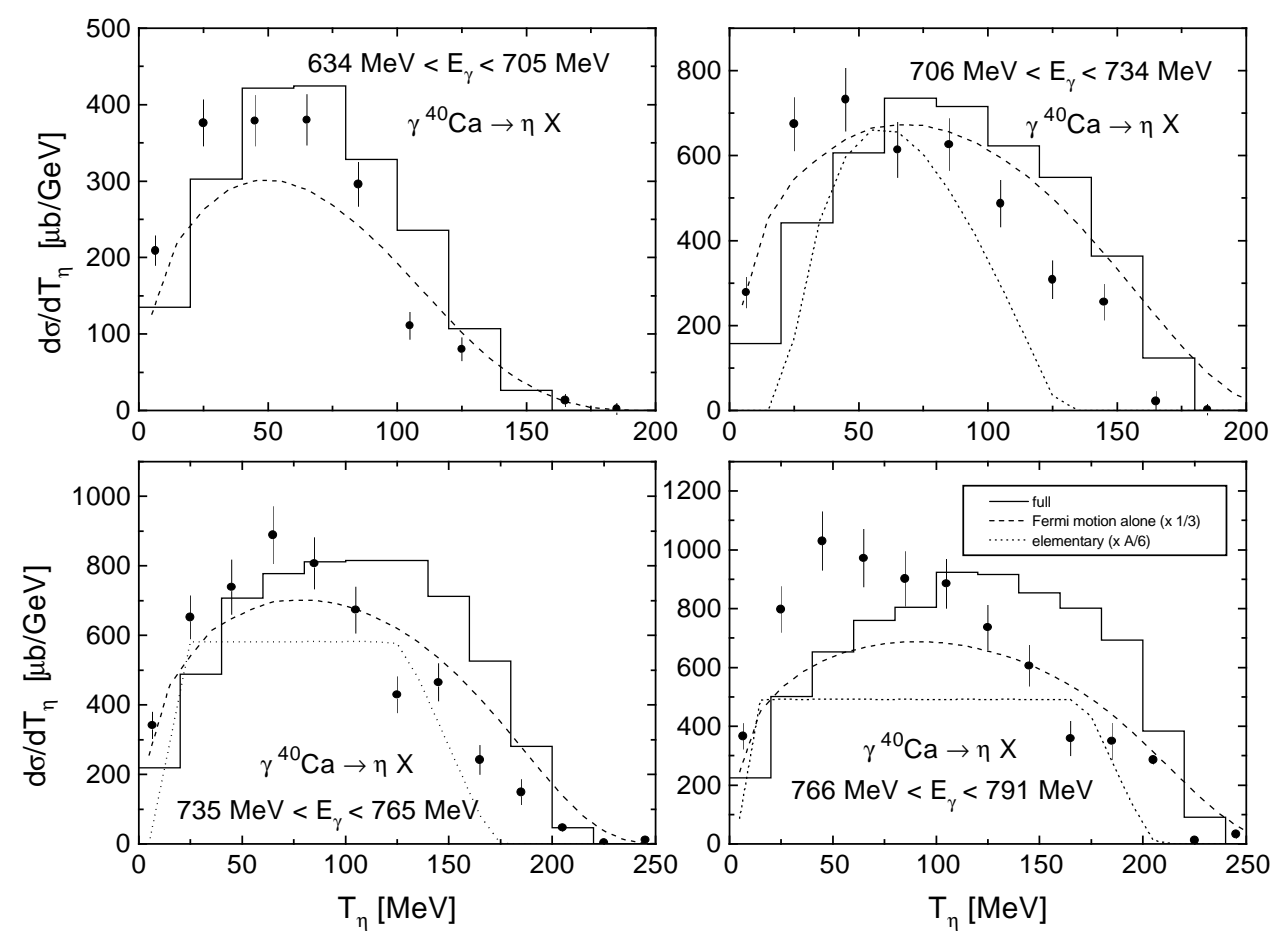

Figure 12: Energy differential eta photoproduction cross sections on ${ }^{40} \mathrm{Ca}$. Explanation see figure 11 . 

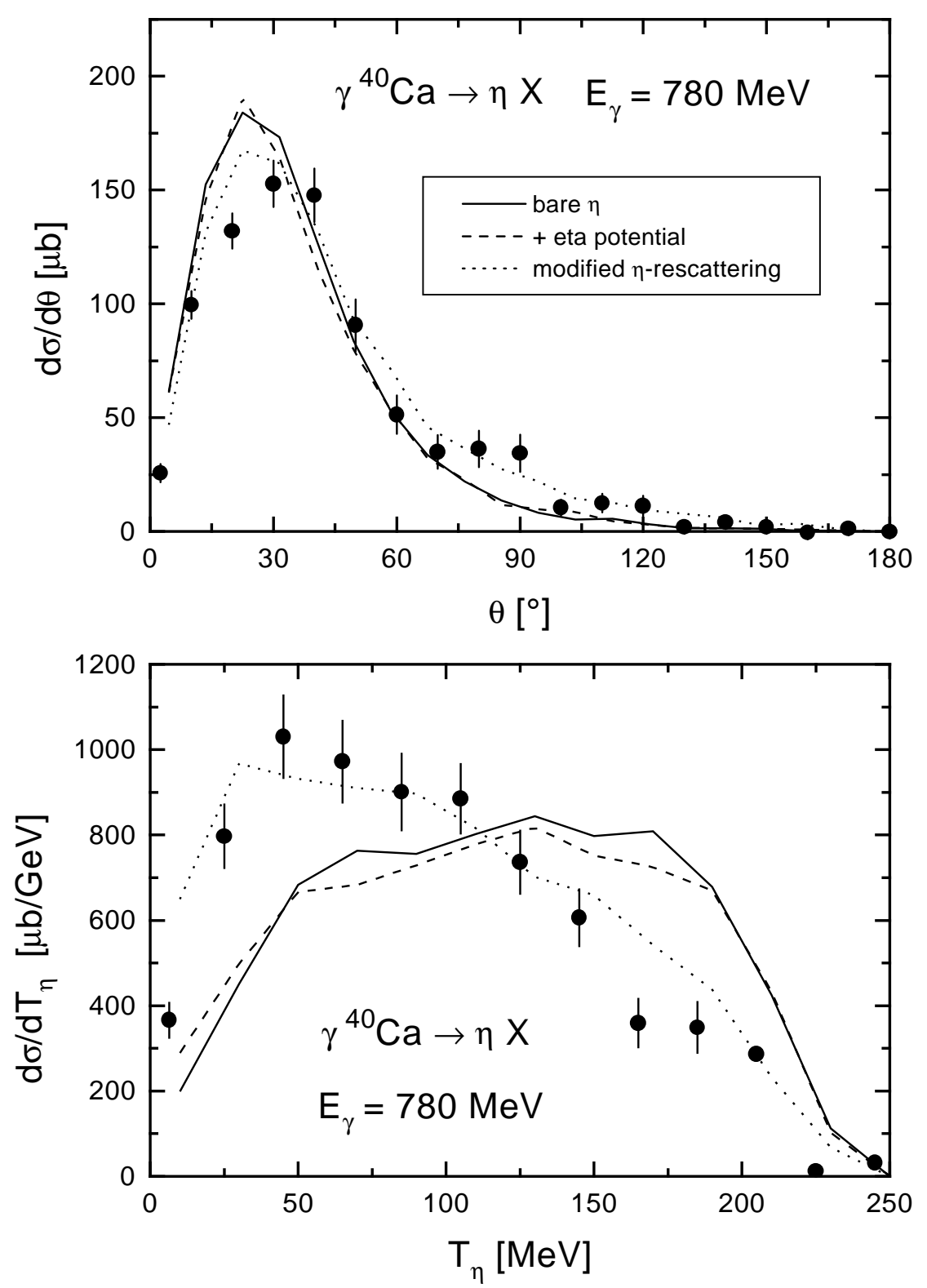

Figure 13: Modifications of the angular and energy differential eta photoproduction cross sections on ${ }^{40} \mathrm{Ca}$. The solid lines represent the previous cross section. Explanation see text. 